\title{
Locating Defects Using Dynamic Strain Analysis and Artificial Neural Networks
}

\author{
L. H. Hernandez-Gomez ${ }^{a}$, J. F. Durodola ${ }^{b}$, N. A. Fellows ${ }^{b}$ \\ and G. Urriolagoitia-Calderón ${ }^{a}$
}

\author{
a Instituto Politécnico Nacional, ESIME SEPI Edificio 5, 3er piso, Unidad Profesional Adolfo López \\ Mateos, colonia Lindavista 07738 México, D. F. México. luishector56@hotmail.com \\ ${ }^{\text {bS }}$ chool of Technology, Oxford Brookes University, Headington Campus, Gipsy Lane Oxford OX3 \\ OBP U. K. jdurodola@brookes.ac.uk
}

Keywords: Dynamic strains, neural networks, location of defects, inverse computing analysis and back propagation.

\begin{abstract}
An inverse artificial neural network (ANN) assessment for locating defects in bars with or without notches is presented in the paper. Postulated void defects of $1 \mathrm{~mm} \times 1 \mathrm{~mm}$ were introduced into bars that were impacted with an impulse step load; the resultant elastic waves propagate impinging on the defects. The resultant transient strain field was analyzed using the finite element method. Transient strain data was collected at nodal points or sensors locations on the boundary of the bars and used to train and assess ANNs. The paper demonstrates quantitatively, the effects of features such as the design of ANN, sensing parameters such as number of data collection points, and the effect of geometric features such as notches in the bars.
\end{abstract}

\section{Introduction}

An engineering problem that has attracted much attention is the location of defects in components and structures. Although destructive and non-destructive evaluation (NDE) methods are routinely used for this purpose there is still scope for improvement in quantitative characterisation of flaws and defects in components. This is helpful for structural integrity analysis. Inverse methods involving application of artificial neural network (ANN) methods can assist with the process.

ANN has been used in recent years to assist in solving mechanical engineering problems. A description of early works on location of defects can be found in a review paper by Yagawa and Okuda [1]. More recently, Achenbach [2] presented a state of the art review of quantitative nondestructive evaluation. He analyzed the application of NDE with ANN and established that a numerical model is helpful for the interpretation of experimental data and the recognition of characteristic signal features.

The aim of this work was to study the use of ANN for locating defects such as voids. The work also considered the effects of parameters such as design of the ANN, locations for data collection, and external geometric features such as notches on the prediction of the location of defects. In order to assess the effect of the parameters, two main geometry cases were studied. A plain bar with a rectangular cross section and a similar bar with an external notch were considered. A notch is a typical feature that may be found on real components and in this study it was included as a simple modification that will have significant effect on wave propagation in the bar. The notch presents a constriction that modifies the magnitude and direction of the stress wave. The additional complexity provides a means to assess the performance of ANN and the potential impact that geometric features that can be found on real component will have on ANN design. Voids were included in the bars in order to simulate the presence of defects. 


\section{Inverse approach}

The inverse approach used consists of several aspects. The finite element method (FEM) was used as the forward solution technique. This was used to obtain strains at selected sensor nodal points when the domain of interest, containing a void defect, was impacted by an impulse load. The strain information obtained at sensor locations was used as the input data for ANNs. The output or target data was the $\mathrm{x}$ and $\mathrm{y}$ coordinates of the centroid of the defects. The inverse analysis for predicting defect location was carried out using the trained ANN. The input and output data sets that were used for training and assessing the accuracy of the network were randomly selected.

The bars studied in the work were modelled with an impulse load at applied one end and assumed fixed at the other end. Several analysis runs for different simulated defect positions were carried out. Five hundred defect locations were considered in total. Strain data for $80 \%$ of the defect locations was randomly selected and used to train the ANN. The remaining $20 \%$ of the data was used to assess the accuracy of the location of the defects and for the evaluation of the effect of parameters such as ANN design, number of sensor points.

\section{Propagation of elastic waves}

When a rectangular section bar is squarely impacted at the end IJ, Fig. 1, longitudinal elastic waves propagate along the length of the bar with a constant velocity provided the cross section is constant and the wavelength is large compared to the lateral dimensions. The velocity of propagation $c_{o}$ is given by equation (1)

$$
c_{o}=\sqrt{E / \rho}
$$

where $\mathrm{E}$ and $\rho$ are respectively, the modulus of elasticity and the density of the material. This equation is valid under elastic conditions assuming that the stress acts uniformly over each section and the transverse sections of the bar remain plane during the propagation. In all the cases analysed in this work, the bar had a small defect that obstructed the waves and hence the resultant transient fields were calculated numerically using FEM because of its versatility to cope with the distortion that will occur to the waves.

\section{Neural network analysis}

An ANN may be described as a non-linear mapping function from multiple input data to a variety of output data. In order to achieve this, a multi layer network has to be trained. When trained, it can produce a good estimate in a short time using only low computing resources.

The computational model simulates layers of neurons that are fully or partially connected. There are no connections among the units of the same layer. In the case of those used in the problem for the work reported, the information propagates in one direction, from the input to the output layer. A neuron obtains information from neurons of the previous layer. All the data arriving at a neuron is processed and a unique output $t_{i}$ is generated based on the relation given in equation (2)

$$
t_{i}=\sum w_{i j} P_{j}+b_{i}
$$

where $P_{j}$ is the input of a previous neuron $j, w_{i j}$ is the connection weight, and $b_{i}$ is the bias. As can be seen in the equation, the neuron merges the weighted values of the inputs and then modifies it by the bias. The resultant value is modified by an activation function before being forwarded to the next layer. For the purpose of this work two types of activation functions were applied, namely the 
tan-sigmoid and linear. The latter was only used in the last layer of the networks. The activation equation of a tan-sigmoid neuron is given by equation (3)

$$
f\left(t_{i}\right)=\frac{1}{1+\exp \left(-t_{i} / T\right)}
$$

where $\mathrm{T}$ is a parameter that determines the slope of the sigmoidal function shape. The network analysis was carried out using the neural network tool in MATLAB [3]. After preliminary test, a network with four layers was selected. The input layer had twenty neurons, while the two intermediate layers had 10 and 5 neurons. A second network with 20-15-10-2 number of neurons in the layers was also considered with the aim of assessing the possible effect of the change of the ANN design on results. In all these layers, sigmoidal neurons were used. The output layer had two linear neurons.

\section{Finite element analysis}

Explicit dynamic finite element analysis was carried out on the bars analyzed. Each element of the mesh had a size of $1 \mathrm{~mm} \times 1 \mathrm{~mm}$. This regular size was possible because of the rectangular shape and dimensions of the bars chosen for the analysis. It should be noted that regularity of element size or shape is not a necessity in the general procedure. The bars were considered thin enough for plane stress elements to be used. Four noded linear parametric representations were chosen for the elements.

An instantaneous impulse step load of $100 \mathrm{MPa}$ was applied at the end, IJ, and the other end, LM, of the bar Fig 1 was modelled as a fixed boundary condition. The Young's modulus, Poisson's ratio and density of the bar were taken as $210 \mathrm{GPa}, 0.3$ and $7800 \mathrm{~kg} / \mathrm{m}^{3}$ respectively.

Strain results were collected at nodes along the line $\mathrm{AB}$ and $\mathrm{CD}$, Fig 1, at $4 \times 10^{-5}$ seconds from the instance of impact at the end of the bars. The number of nodes used for the collection of strain data was varied as noted in the results section below. An elastic wave travelling from the left end of the bar will have interacted with the defects included as voids and reached the output sampling nodal positions.

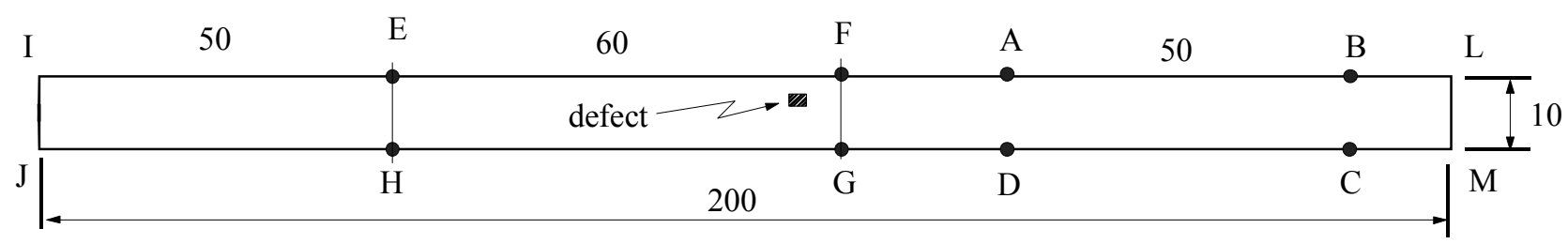

(a)

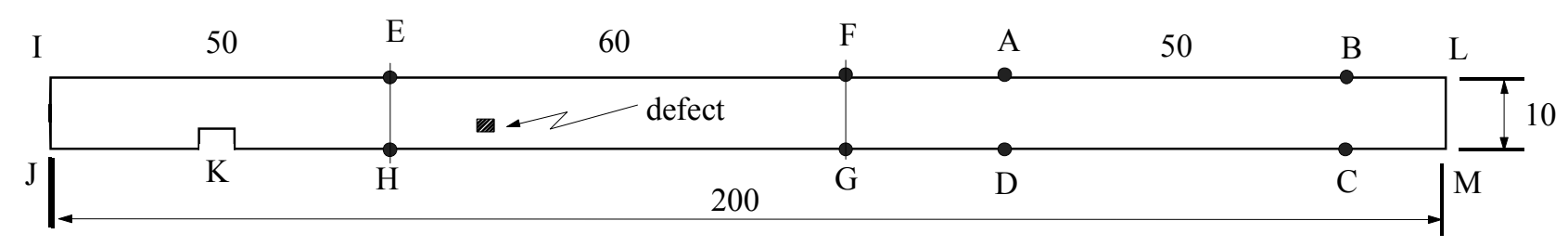

(b)

All dimensions in $\mathrm{mm}$

Figure 1 Sketch of bars analysed (a) plain bar (b) bar with notch 


\section{Results and discussion}

Two major cases were considered in the study. In one case, a plane bar was considered and, in the other case, the plain bar had a notch included at one end as illustrated in Fig 1. The effects of ANN design and the number of output sampling points were studied.

\section{Plain bar analysis}

Finite element analysis was carried out as highlighted in the foregoing. Five hundred element locations in the region EFGH, Fig 1, were damaged in turns and the effect of each damage location on axial strain results was collected from the edges $\mathrm{AB}$ and $\mathrm{CD}$. Three cases with varied number of nodal data collection were considered, these were 8,5 and 2 nodes on each of the edges $\mathrm{AB}$ and CD.

In the study carried out, $80 \%$ of the damage location data was randomly selected and used for the training of the ANN and the remaining $20 \%$ was used to assess the network. Strain data was used as input to the ANN and the $\mathrm{x}$, $\mathrm{y}$ coordinates of the centroid of the damaged element were used as the target. Representative results obtained for predicted location of defects and actual locations are plotted for ten out of the one hundred locations in Fig. 2.

It can be seen from the results in Fig 2 that very good agreement was obtained between actual location and predicted location of defects in the bar. This is not totally surprising as the wave-front is plane and its location from the impact end is simply linearly related to time from initial impact. There are still no precise and widely accepted algorithms for the choice of number of layers of neurons and number of neurons to be used in the different layers in order to solve a problem. In order to attempt to check the sensitivity of results to the design of the ANN, a second network design was considered apart from baseline network used in the study. Both networks had four layers and the following number of neurons in the four layers 20-15-10-2 and 20-10-5-2. The major difference in the designs is that the latter has five fewer neurons in each of the intermediate layers than the former. The number of neurons will have a significant effect on the computational resources required to solve a problem especially in large networks. There were no significant differences in the results of the two networks considered in this study. This suggests that both networks are adequate and that the baseline network had more than necessary neurons. There is scope for further trimming down although this was not carried out in this work.

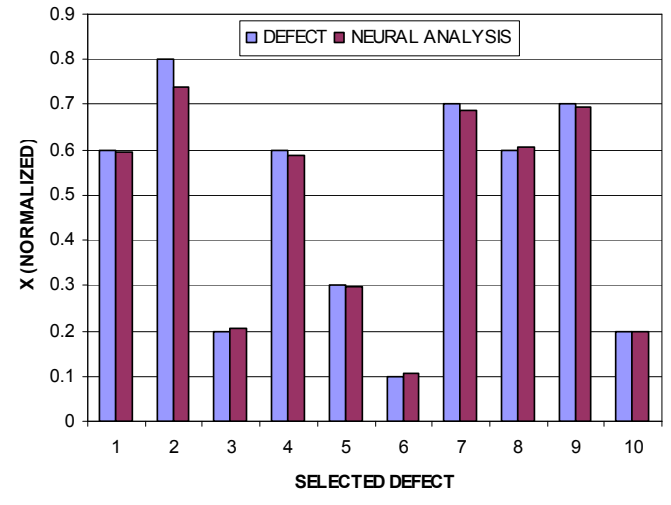

a)

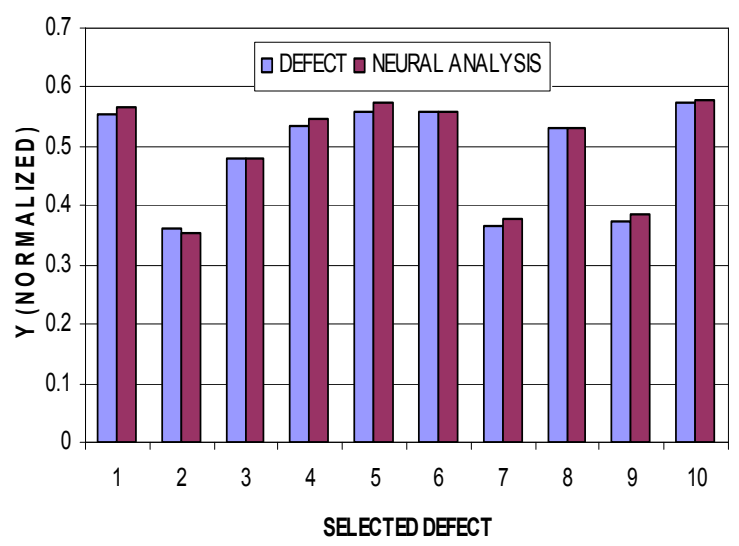

b)

Figure 2. Comparison between predicted and actual coordinates of the defect, (a) X coordinate (b) $\mathrm{Y}$ coordinate for the bar without notch using five sampling points each, on edges $\mathrm{AB}$ and $\mathrm{CD}$, Fig 1.

Another interest in this work was to investigate the effect of the number of sensor points used in collecting data for the training and assessment of the ANN. In a practical application, the fewer the 
number of sensors the better because the processes involved in the detection of defects will be made easier. The sensors used in this study had to be placed downstream relative to the position of the defects. This is necessary in order for the travelling wave to be modified by the effect of the presence of the defect before reaching the sensor locations. When 8 nodal sensor points was considered on each of the sides $\mathrm{AB}$ and $\mathrm{CD}$, Fig. 1, every defect was located within $2.5 \%$ of the dimension of the coordinate of the defect. For the cases of five nodes and three nodes used on each of the sides, the accuracy of the location dropped to $6 \%$ and $15 \%$ respectively. The number of sensor nodes used in this work is fewer than those typically quoted in the literature [Ishak et al [4]]. The results obtained show that there is still scope in developing guidelines for determining the minimum number and location of sensor points for accurate and effective detection of defects.

\section{Analysis of bar with a notch}

This study considered the same bar as that in the last section above, except that a notch was introduced in position $\mathrm{K}$ as illustrated in Fig. 1. The notch has a dimension of $4 \mathrm{~mm}$ by $3 \mathrm{~mm}$. As indicated in the introduction, the notch has the effect of modifying the magnitude and direction of the stress wave. The position of the notch has effect on the actual strain values that will occur at any particular location downstream from the notch. The aim of the location of the notch in this study was to place it as near as possible in the path of the travelling wave in order to maximise its deflection effects. The notch was also placed far enough from the impact end to avoid possible interaction with the end effect. The location in this particular study was chosen to be about oneeighth of the length of the bar, measured from the impact end. It should be noted many other locations of the notch could have a similar effect. Further investigation of the effect of the precise location of the notch in relation to other features in the bar is deferred until further studies. The analysis procedure and parameters considered were identical to those in the previous section. The representative results for prediction and actual location of defects are shown in Fig. 3.

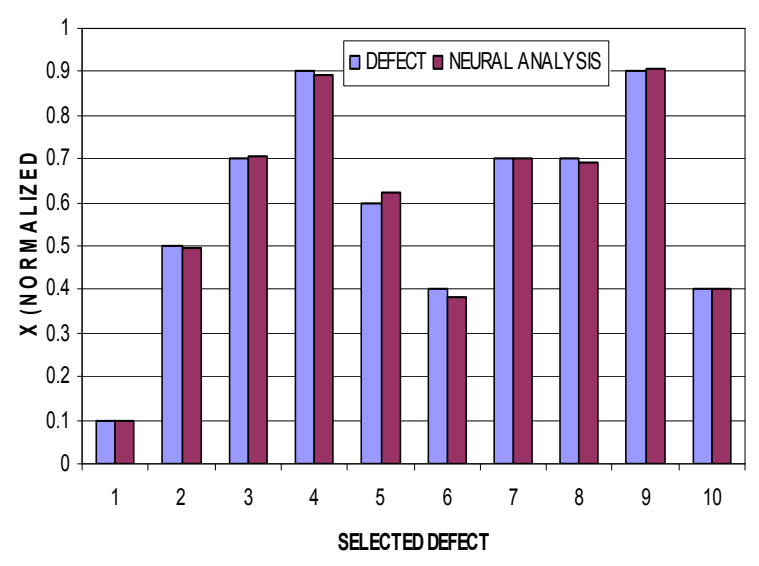

a)

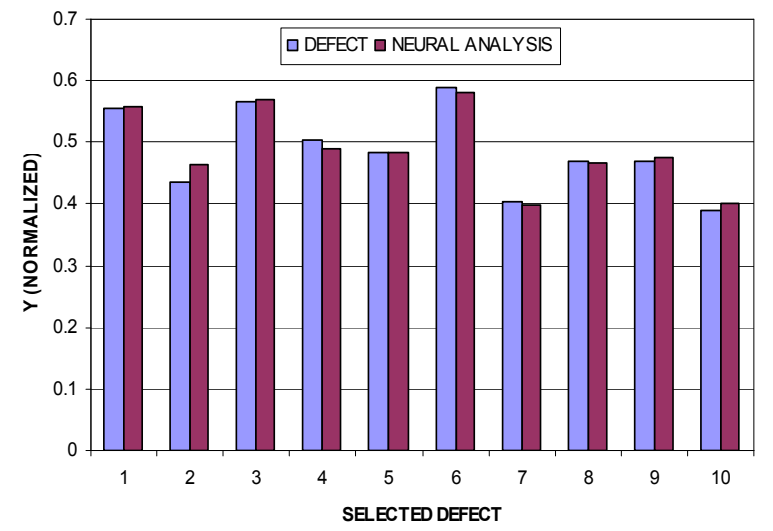

b)

Figure 3. Comparison between predicted and actual coordinates of the defect, (a) X coordinate (b) $\mathrm{Y}$ coordinate for the bar with notch using five sampling points each, on edges $\mathrm{AB}$ and $\mathrm{CD}$, Fig 1.

Very similar results and accuracies were obtained in this case as in the case of the bar without the notch. As in the last case, the results demonstrate that the two ANN designs considered are adequate for the analysis of the problem. This shows that a four layer network system with the number of neurons chosen is able to resolve the effects of the distortion of the wave as well as the encoded effect of the defects accurately. 


\section{Conclusions}

An ANN based defect localisation procedure has been presented and the effects of ANN design and number of data sampling points are highlighted. This preliminary study showed that very accurate results can be obtained even with relatively few data sampling points. Localisation achieved was up to $2.5 \%$ of the actual dimensions of the coordinates of the centroid of the defects. Variations in the number of intermediate neurons considered did not alter the accuracy of predictions significantly even for the case of the bar with a notch. This demonstrates the versatility of ANN method and that there is scope for further reduction of the number of neurons used in the analysis carried out without necessarily losing accuracy. The results obtained show that the effect of the number of sensor points used on the accuracy of predictions is as to be expected very significant. There is therefore further scope in developing guidelines for determining the minimum number and location of sensor points for accurate and effective detection of defects.

\section{Acknowledgements}

The first author kindly acknowledges the support given to him during the development of his sabbatical project by IPN, COFFA, CGPI and Oxford Brookes University.

\section{References}

1. Yagawa, G. and Okuda, H. (1996) Neural networks in computational mechanics. Archives of Computational Methods in Engineering. Vol.3, 4, 435-512.

2. Achenbach, J. D. (2000) Quantitative non-destructive evaluation. International Journal of Solids and Structures. 37, 13-27.

3. Demuth, H. and Beale, M. (2001) Neural Network Toolbox for use with MATLAB, The MathWorks

4. Ishak, S. I., Liu, G. R., Shang, H. M. and Lim, S. P. (2002) Non-destructive evaluation of horizontal crack detection in beams using transverse impact. Journal of Sound and Vibration. 252(2), 343-360. 